World Maritime University

The Maritime Commons: Digital Repository of the World Maritime University

\title{
Lifelong learning: the 21st century skill to guide maritime training and development
}

Angelica Sogor

Hill Robinson Yacht Management

Follow this and additional works at: https://commons.wmu.se/imla2021

Part of the Education Commons

\section{Recommended Citation}

Sogor, A. (2021). Lifelong learning: the 21st century skill to guide maritime training and development. In Pazaver, A., Manuel, M. E., Bolmsten, J., Kitada, M., Bartuseviciene, I. (Eds.), Proceedings of the International Maritime Lecturers' Association. Seas of transition: setting a course for the future (pp. 30-40). World Maritime University. http://dx.doi.org/10.21677/imla2021.02

This Paper is brought to you courtesy of Maritime Commons. Open Access items may be downloaded for noncommercial, fair use academic purposes. No items may be hosted on another server or web site without express written permission from the World Maritime University. For more information, please contact library@wmu.se. 


\title{
Lifelong Learning: The $21^{\text {st }}$ Century Skill to Guide Maritime Training and Development
}

\author{
Angelica Sogor \\ Senior Yacht Manager, Hill Robinson Yacht Management, Fort Lauderdale, USA, \\ ang.sogor@gmail.com
}

\begin{abstract}
Each educational institution, each industry, and each business sector within an industry is likely to have their own unique requirements for what 21 st century skills are needed to develop a successful, innovative, and sustainable future. When looking at a few areas (including educational and professional contexts) where research and strategy development have been conducted on this matter, there are some common trends. One of these common trends is the ability to understand, appreciate, and act on the concept that learning, personal growth, and professional aptitude are not "one and done" achievements. In order to develop a core set of professional skills, a transferable set of general skills, and a dynamic set of $21 \mathrm{st}$ century skills, it is imperative that one first develops the most critical skill of being a lifelong learner.
\end{abstract}

This paper focuses on: what it means to practice lifelong learning; how a practice of lifelong learning can bridge generational gaps within the maritime industry; and how a practice of lifelong learning can develop shipboard mariners and shoreside maritime educators who adapt to and lead technological and innovative industry growth.

Keywords: lifelong learning; 21 st century skills; maritime education and training; professional development

\section{Introduction}

Searching for a list of critical " $21^{\text {st }}$ century skills" produces a laundry list of online results. Often, the lists are categorized by target audience - the $21^{\text {st }}$ century skills needed for students, teachers, and other working professionals. The National Education Association (n.d.) posits that $21^{\text {st }}$ century learning for American schools is comprised of the following focal points: "emphasize core subjects; emphasize learning skills; use $21^{\text {st }}$ century tools to develop learning skills; teach and learn in a $21^{\text {st }}$ century context; teach and learn new $21^{\text {st }}$ century content; [and] use $21^{\text {st }}$ century assessments that measure core subjects and $21^{\text {st }}$ century skills."

A contributor to The Rand Blog recognized that the acquisition of $21^{\text {st }}$ century skills often requires a more tailored approach than a "one size fits all" approach, which is often dictated by one-way lectures and use of textbooks. In this familiar approach, one lecture and one textbook are expected to equally reach many students, backgrounds, learning levels, and learning styles. This strategy has evolved, but is still common in both teaching children and adults. In order to more appropriately impart $21^{\text {st }}$ century skills, however, nine methodologies are suggested to offer a more dynamic approach. Included in the list of nine methodologies are: "make it relevant; encourage transfer of learning; teach students to learn to learn (metacognition); 
exploit technology to support learning; [and] foster students' creativity" (Saavedra \& Opfer, 2012).

With some understanding of what $21^{\text {st }}$ century skills students need to learn and how teachers can more effectively facilitate this learning process, one can begin to paint the picture of how educational systems have evolved, and should continue to evolve, to meet the demands of a modern and ever-changing society. Moving beyond the traditional and formal educational system: what does the skill set look like for that society, for those who are not enrolled in school and for those whose profession is not directly involved with educational systems? What are the $21^{\text {st }}$ century skills for a typical working professional?

A Forbes contributor identified four generalized skills that have relevance, vast applicability, and can be transferred across various professional industries and roles. The skills are "critical thinking, collaboration, creativity, and communication" (Quast, 2014). It likely does not take long to consider how those four generalized, core skills can be applied across different professional functions and industries. Critical thinking and creativity contribute to both dayto-day problem solving and development of long-term strategies for business development. Meanwhile, collaboration and communication foster even the most rudimentary internal professional processes, while also being integral aspects to more complex external marketing, partnerships, and customer service.

Looking more closely to the specific $21^{\text {st }}$ century skills and qualities required for the maritime industry, the Maritime College at the State University of New York highlighted strategies as an educational institution for preparing students and faculty for the future. Their strategies to achieve this included focusing on "...a strong technical foundation, applied learning opportunities, and leadership development... [and] ... lifelong learning that enables [students] to meet the future in leadership roles that positively impact society" (SUNY Maritime College, n.d.).

One can begin to picture, with more clarity and detail, how skills such as critical thinking, creativity, collaboration, and communication (Quast, 2014) are crucial to onboard operations in the maritime domain - as well as in the myriad of shoreside functions (operations, resource management, regulatory compliance, training, and emergency preparedness - to name a few) that govern and support onboard operations. For example: communication and collaboration are needed to support both routine operations in familiar ports and emergency response efforts, where unfamiliar port agents and authorities may become new and necessary resources.

Outside of the United States, the European Marine Board is an organization focused more predominantly on marine science and research. In recognition of their role in supporting the European Commission's Blue Growth Strategy, the European Marine Board reviewed training and education in both the marine and maritime industries in their Future Science Brief titled "Training the $21^{\text {st }}$ Century Marine Professional." In this brief, they identified nine "key targets for marine graduate training in the $21^{\text {st }}$ century," which included "encourage international networking and collaboration; incorporate innovative training methods; [and] facilitate continuous professional development" (Vincx et al., 2018, p. 5).

From students to teachers to non-academic industry professionals to the specific operations of the maritime industry, there exist many choices when trying to narrow down and identify key skills required for evolution within society in the $21^{\text {st }}$ century. Each of these important skills are not commodities that can be acquired overnight, nor are they skills without a need to be 
reviewed and refined. One must dig deeper to determine not only how these skills are acquired, but also how they are maintained and how they are able to evolve across industries, functions, and technological changes. In this pursuit to dig deeper, it is hypothesized that the skill of lifelong learning is the skill that must be acquired before choosing to develop a core set of professional skills, a transferable set of general skills, and a dynamic set of 21 st century skills.

\section{What is Lifelong Learning?}

Lifelong learning can be defined most simply as learning that: "take[s] place at all stages of life cycle (from the cradle to the grave) and, in more recent versions that it should be life-wide; that is embedded in all life contexts from the school to the workplace, the home and the community... [it] is the continuous building of skills and knowledge during one's life" (Laal, 2011, p. 471).

Beyond the continuous acquisition of increased knowledge and skills, lifelong learning can be distinguished from other pursuits that are finite, pre-determined, or infrequent by examining one's attitude or motivation. Lifelong learning is not merely an education or defined activity to earn a certificate, enhance one's application for promotion, or (literally or metaphorically) complete a required or expected checklist. Lifelong learning is observed through constructive and questioning attitudes, self-derived or intrinsic motivations, and a personal willingness that is present in a variety of social, educational, cultural, and professional environments. The process of lifelong learning is dependent on active, planned, and intentional engagement from an individual (Smith \& Spurling 1999: 9). As Vincx et al. explain, effective lifelong learning must be facilitated both by an individual's environment and their own engagement: "Whereas most employers recognize the importance of the need to have a workforce that is continually developing its skills, knowledge and competencies, individuals share the responsibility, and as such need to pro-actively engage in their own personal and career development and lifelong learning" (2018, p. 35).

The definition of lifelong learning (as described in the two previous paragraphs) is simple, but vast and diverse - the pursuit of learning is often compulsory or compliance-driven, but to truly exemplify the principles of lifelong learning, the lifelong learner reaches far beyond required education. The lifelong learner pursues formal learning, recognizes opportunities to learn from others even in day-to-day activities, and seeks out learning opportunities throughout their lifetime, without prescribed oversight and "beyond the formal structure of an educational institution" (teAchnology, n.d.).

The lifelong learner is often the person asking the "why" behind decision-making processes, the person asking "what more" can they do beyond a task or role they have completed or become complacent in, and the person challenging the status quo to consider "what if" operations were conducted differently.

Why is lifelong learning important? Why is it not enough to know and be satisfied with the skills needed for today's society, for today's economy? What is the value in continuing to learn when one has already completed educational or professional milestones?

One of the easy-to-understand reasons that demonstrate the importance of lifelong learning is considering the reality that today's society and today's economy are not the society and economy from $10-20$ years ago, and they will not be the society and economy $10-20$ years from today. Simply: today's skills are not enough if one wants to remain an essential and 
relevant member of society's workforce. The most visible, the most undisputable way to understand and appreciate how relevance can be temporary is considering the impacts of machinery, automation, and technology across industries and countries. As highlighted by one contributor to Forbes: "With rapidly changing technology, the speed at which businesses must operate to be competitive in a global economy and with human life expectancy rising steadily, it's clear that lifelong learning will become essential for humans to remain relevant in the workplace." More specifically, the impacts of evolving technology remain even if human functions are not outright replaced by technology because "...the shelf-life of technical skills, such as software development, are getting shorter and shorter." Predictions suggest "...more than 120 million people will need up-skilling or re-skilling in the next three years and that the average length of training needed to close skills gaps has increased from 3 days to 36 days in just five years' time" (Busteed, 2020).

The benefits of lifelong learning, of continued engagement, of active pursuits to master something new extend outside of professional realms and business operations as well. Specifically when considering generational gaps in professions, it may be easier to think older or more experienced persons will not see similar benefits from lifelong learning, especially for those who are actively planning career transitions or retirement. Lifelong learning, however, is not merely about marketing oneself for a future promotion or about achieving job security in a dynamic and evolving future.

In an article highlighting various benefits of lifelong learning, a contributor to the Harvard Business Review recognized that learning pursuits and goals are not static over a lifetime. Coleman (2017) stated: “As we age, though, learning isn't simply about earning degrees or attending storied institutions. Books, online courses, [massive open online courses], professional development programs, podcasts, and other resources have never been more abundant or accessible, making it easier than ever to make a habit of lifelong learning. Every day, each of us is offered the opportunity to pursue intellectual development in ways that are tailored to our learning style." Coleman (2017) listed several key benefits or outcomes that occur when such learning opportunities are pursued, including: increased economic earnings, reduction in stress, cognitive benefits, enhanced social and interpersonal interactions, and the "electrifying" and "worthwhile...expression of what makes every person so special and unique."

When considering the personal and professional benefits of lifelong learning and understanding what distinguishes lifelong learners from other learners, one can begin to understand why lifelong learning is the underpinning needed to develop and sustain other $21^{\text {st }}$ century skills. Collaboration and communication (Quast, 2014) are not only important in the contexts of human-to-human interactions, but they must be adaptable for how technology (e.g., smart phones, video conference calls, webinars, and shared software tools) has changed human-tohuman interactions. It is hardly enough to have comprehension of today's collaborative tool; one must embrace and adapt to tomorrow's tools to maintain personal and professional connections and communications.

Furthermore, there are many human-to-data interactions that proliferate across industries. People need to process, respond to, and even manipulate the data they receive from various technologies and systems that are integral to their job or their industry - whether thinking of the advanced coder who applies computer programming language to design a vessel's planned maintenance system or thinking of the end user who received routine data and non-routine error messages from that software. Learning requires the coder to design the software one time or requires the end-user to learn how to use the software as initially installed. Lifelong learning 
enables the coder to respond to user feedback to fix bugs and provide more explanatory error messages and enables the end-user to adapt their understanding of the software with each version released.

The maritime industry is rife with educational and certification requirements, as well as shipboard and shoreside technological advancements. These aspects create ample opportunities not just for required learning and promotional growth, but also for lifelong learning. How can this lifelong learning help reduce generational differences, develop adaptable mariners, and encourage advancements in maritime training and education?

\section{Applications of Lifelong Learning in the Maritime Domain}

In the maritime domain, mariners (or aspiring mariners) can be taught navigational skills, how to perform effective Bridge Resource Management (BRM), how to apply leadership and managerial skills, and how to use shipboard technologies, such as Radar and Electronic Chart Display and Information System (ECDIS). Individual ship owners or managers can: further train their shipboard employees in unique company policies and procedures, focus voyage planning in the specific routes and regions of the ship's expected operations, tailor shiphandling classes to anticipated ports of call, and collaborate with manufacturers to ensure that mariners know and understand the specific types of safety systems and environmental technologies equipped on their vessels. Training may seem endless at times; even after attaining the rank of Captain, various trainings and certifications require periodic renewal and shipboard systems may be frequently replaced with newer versions or completely new systems. Whether driven by industry regulations, company requirements, or an individual's desire to be promoted, there are regular opportunities for on-going training and development for mariners.

With all these training schemes already in place, it may seem that there is hardly a need for one to be a lifelong learner; surely, they must become one by default when they pursue such a highly-regulated industry or have goals of professional growth. These factors alone, however, do not create a lifelong learner. One can receive training in how to lead and manage a team, but one cannot necessarily receive training in wanting to lead and manage others effectively. One can receive an updated instructional manual and guidance from a manufacturer regarding the latest ECDIS software update, but one is not necessarily provided with the optimism, engagement, patience, and persistence that are often required to "re-wire" the technical knowledge of the previous version, onto which they are consciously or unconsciously holding.

The qualities and characteristics of lifelong learning are then what separate those who simply find themselves in regular training courses, versus those who may receive regular training but also appreciate and seek out the opportunity to make that training an active and engaging learning experience beyond acquisition of a particular skill and beyond only the present moment of receiving training. In other words: while most technical skills can be taught and a license or certification may document such skills, it is often difficult to teach or instill the qualities of wanting those skills, wanting more than those skills documented on paper, and wanting more than what is required for that paper or ultimate professional position. This is where lifelong learning not only separates obligatory trainees from engaged learners, but also where lifelong learning can reach out and expand its roots to improve mariners and maritime educators far beyond standard industry and technical requirements. Lifelong learning is not only the underpinning for various other $21^{\text {st }}$ century skills, it is an underpinning that can transform the way the maritime industry sustains current and future generations, across varying professional functions. 


\section{Bridging Generational Gaps}

While learning is hardly ever "one size fits all," younger generations or those newer to the industry may require more formal training, with typical instructor-led courses and hands-on classroom or shipboard activities. At the same time, younger generations may also be more apt to learn and engage with learning technologies or even through platforms initially designed for social media and personal communications. For example: Generation Z (those born between 1995 and 2000) prefer digital and contemporary experiences, often foregoing personal contact by primarily choosing to engage in communication through technology (Jurenka et al., 2018).

Because these types of technological mediums are pervasive in the day-to-day life of younger generations, opportunities for learning may not even appear as such when they occur informally or with tools typically used for social or entertainment purposes. Alternatively, older generations who may have grown up with very structured "lecture and learn" strategies, facilitated exclusively through instructor-led classroom training, may find traditional, structured trainings something that they have "grown out of" or have surpassed the need for, as their professional rank and credentials expanded. They may have some engagement with social and other technologies, but may not see these as educational or informative tools.

Lifelong learning can aid in bridging some generational gaps if multiple generations are open to engaging with one another across technology platforms. Examples of learning and engagement with modern technology include: "how to" videos hosted on YouTube; interactive polls or quizzes shared through Instagram's "Story" feature; and a discussion of industry events shared in a LinkedIn group. Finding a technological platform that is compatible and userfriendly across different types of devices (traditional desktop computer, tablet, and mobile phone) can bring different generations together on the same platform - even when they work in different professional areas, are on different ships within the same company, or are working within the same company but some are actively sailing and some are on vacation.

Perhaps now more than ever, such technological spaces also offer a connectedness, that expands beyond formal and informal learning, through a professional community that support the well-being of seafaring professionals. As Buchmann (2021) explained the role of technology in aiding seafarer's mental health, particularly in response to the COVID-19 pandemic, he stated: "Providing personal internet access as a way to keep in touch with people can be a lifeline for seafarers at sea for months. Being able to send emails or do video calls is something that more shipping companies are recognizing is a way to tackle mental health issues." Sharing photos, hearing stories, discussing articles, and taking interactive quizzes with colleagues through social media and learning technologies does not substitute for video calls with friends or family. However, these actions can offer another pathway for staying socially connected and contributing to mental wellbeing for seafarers, all while also offering an opportunity to engage in lifelong learning.

Creating this space to share, discuss, and receive information in different formats (e.g.. text, photo, audio, video) and interact (e.g., like, comment, vote) can foster some of the collaboration, creativity, and communication that were highlighted by Quast (2014) as key professional skills, which are valuable across industries and professions. In order for such a platform to be truly informative, interactive, and purposeful, those engaging with the platform have to want to actively provide and receive those qualities; it is not enough to train someone how to use any company-specific tool or company-specific application of a publically available technology. 
When learning, collaboration, and communication technologies are effectively employed and users are actively engaged in these tools, these platforms "have the capacity and capability to bring expertise to a large audience of professionals at all career stages within an international context (subject to availability of high-quality internet connection)" (Vincx et al., 2018, p. 35).

Sometimes these platforms, such as e-learning systems, provide evidence of completed training through a course completion certificate. In other, more informal platforms: there is no certificate of completion provided after one joins the platform or after a specific number of days they are active in the platform; there is only the resulting joys of practicing lifelong learning, such as increased engagement in their professional endeavors, enhanced social experience and connection with colleagues, and increased adaptability to share and gain new knowledge in new environments.

Bridging generational gaps is also accomplished through knowledge sharing. This objective can be achieved through technology, but it is not required to occur over a specific mode or platform. While different generations are actively employed together, it may be assumed that those with greater experience levels are teaching, leading, and coaching their younger or greener colleagues. Sometimes, however, it is not until an experienced professional leaves their employment (either by transitioning to a new career or by retiring) that a company recognizes the gap in knowledge and process that occurs without that experienced professional. Further, in some instances, this may be the only time it is realized that the prior assumption (of experienced professionals teaching, leading, and coaching others) was more of an unspoken aspiration and less of a planned reality.

It is possible, however, to transform such an aspiration into reality. A company can develop written procedures and processes to ensure implicit knowledge of experienced employees becomes explicit knowledge for others. A company can also offer internal professional development, mentoring programs, and even casual networking or socializing events. Through these and other methods, a company can create a culture where learning and knowledge sharing is not exclusive to a single training course or specific company procedure; learning is part of a company's daily operations and interactions. In this culture where active engagement is encouraged across different strategies, a company can promote the development of its employees not only as professionals, but as lifelong learners who routinely strive to connect with, collaborate with, learn from, and share something new with their professional peers. This professional culture and its lifelong learners then actively bridge generational and knowledge gaps as routinely as they reply to emails. Over time, this culture can significantly minimize the potential loss of knowledge and leadership when employees depart the company.

\section{Developing Mariners}

Many mariners sailing in the deck and engine departments aspire to achieve the required licensing, certification, and professional experience to sail as Captain or Chief Engineer, respectively. As previously highlighted, there is no shortage of training for mariners with these professional goals. Sailing in these ranks, however, should be about more than having the responsibility and authority to make strategic or tactical decisions, oversee maintenance and equipment, and ensure compliance with all required health, safety, environmental, and security regulations. Commanding a ship's department (or the ship in its entirety) should also entail leading and motivating others through displays of lifelong learning, such as encouraging questions and transparent discussions regarding operations and decision making. 
When lifelong learners attain these leadership positions, they have often already exemplified strong critical thinking, collaboration, and communication skills, in addition to technical knowledge and skills. They may continue to seek opportunities to ensure their skill set is upto-date with the latest technologies and equipment outfitted on a ship, and thus continue their function as a lifelong learner. Ideally, however, those in command are not only engaging in their own lifelong learning, but they are also supporting their onboard teams and shoreside colleagues in engaging in lifelong learning. When lifelong learners flourish and grow into leadership positions, they often want to share their passions and motivations with others. Whether they formally act as a mentor to a cadet or informally impart some local knowledge of the next port of call in a casual conversation over coffee on the Bridge, these leaders can use their collaboration and creativity to encourage the development end engagement of the mariners they lead and manage.

While mariners will often have the opportunity to gain new technical skills in a classroom or simulation, there is also opportunity for them to learn through: listening to their colleague's sea stories in the crew mess, achieving understanding through practicing closed-loop communications between the Bridge team and Pilot, and actively participating in a drill debrief to identify what team actions could have been better or executed differently. Encouraging mariners to apply technical skills in real-life applications, maintaining training and certifications that are up-to-date with technology advancements, and asking questions are important for professional development at all career levels - and these actions are not, and should not be, limited to formal classroom training. Those charged with formal command, as well as those who may have informal leadership roles, should be tasked with exercising their own lifelong learning goals while also actively shaping the current and future generations of mariners to develop similar lifelong learning goals. Practicing lifelong learning enables mariners to support their individual value in a dynamic society; and encouraging lifelong learning in others enables the maritime industry as a whole to adapt to global, economic, and technological demands that are larger than any single ship or company.

\section{Developing Maritime Educators}

When discussing the development of mariners, the component of their formal shoreside education and training must also be considered. And surely, these students and trainees cannot be expected to develop or sustain $21^{\text {st }}$ century skills if their educators and trainers are absent of such skills. Many educators find themselves teaching others because it is a passion for them to share knowledge and it is fulfilling to inspire future generations. Others find themselves in such roles because of circumstances outside of their control that no longer allow them to actively sail on ships. The transition to an instruction-based role allows them to maintain a connection with the seafaring community, to which they have strong professional and personal ties. All educators, regardless of their initial reason for entering an instructional role, must strive to support and inspire the development of lifelong learners. Instructors can achieve this, in part, by: encouraging students to find real-world applications of course material; motivating students to make connections and draw relevance for course material beyond today and beyond their present role; encouraging students to ask questions and share their experiences; and adapting course material to include current events, recent case studies, and advancements in technology.

In order for maritime instructors and educators to truly and organically create lifelong learners who are readily able to absorb and apply various $21^{\text {st }}$ century skills, these instructors and educators must be lifelong learners themselves. They can practice lifelong learning by: seeking out new instructional strategies and tools to present content in more effective or engaging ways; 
respecting and appreciating the experience and knowledge students bring to a course regardless of background and experience level; collaborating with peers when opportunities for interdisciplinary projects arise; and retaining a recognition that, despite their subject matter expertise, their individual development (as a person, as a leader, as an instructor) is a continuously evolving process.

When reviewing the European Marine Board's vision for education and training programs, and picturing young and new professionals who are "... applying multi-disciplinary knowledge to address complex marine and maritime issues which cut across scientific, environmental, economic and social systems," one can wonder how graduates and professionals will be able to achieve such knowledge and appreciation for the dynamic and inter-connected maritime domain if their training and education does not expose them to such a domain (Vincx et al., 2018 , p. 29). When picturing instructors who stick too rigidly to a pre-defined curriculum, do not engage in back-and-forth discussions, and provide reference material or examples that are limited to only specific scenarios and specific geographic regions, it is almost impossible to imagine being able to contribute to the development of students or professionals who "...develop innovative approaches that emphasize not only multi-disciplinarity, networking, and collaboration across sectors, but also create links between different areas of expertise and skills" (Vincx et al., 2018, p. 29).

When considering the unique skills required for the international maritime industry, one would not anticipate to receive training in shiphandling, meteorology, crisis management, or engine room resource management from one who possesses no discernable skills or qualifications in the respective subject matter. Similar to those industry-specific skills: when considering more general and transferable skills that enable critical-thinking and problem-solving, facilitate strong interpersonal relationships, and demonstrate openness to new technology, one would anticipate that training or practice in applying these skills would also be led by one with relevant skills or qualifications. Therefore, if seeking to employ lifelong learning in professional settings and shipboard environments to bridge generational gaps and develop multiple generations of mariners, it is required to also look for maritime educators and instructors (whether they are based in a traditional classroom or have adapted to technological platforms) to exhibit the same lifelong learning qualities sought after in maritime students and professionals.

\section{Conclusion}

When looking beyond technical skills required for a singular role or function in the maritime industry, it is important to consider that the industry is comprised of: many roles and functions across ship and shore, professionals who may have begun their first career in another field, and many different cultures, nationalities, and geographies. Company procedures, recruitment specialists, and hiring managers can sometimes narrow down how to define an ideal professional, regardless of their individual characteristics and experience, by identifying industry-specific skill sets believed to be required to for success and achievement. But the consideration, the evaluation, the qualifying factor for success and achievement cannot be limited to only the skill set needed today. The engagement, motivation, and attitude of a professional must also be taken into consideration because these professionals will not all stay in that singular role or function for the entirety of their career and because the technologies, regulations, and environment that guides the industry will not be static in the entirety of any one person's career or one company's business plans. To fill the gaps that exist between today's measure of success and the future's needs, companies and industries can continue providing 
training, providing leadership and promotional opportunities, providing collaborative networking events, and implementing new systems and equipment that out-pace outdated ones.

Providing training, opportunities, events, and equipment sounds like a robust plan, but it may not be enough to create or sustain professionals who require $21^{\text {st }}$ century skills to achieve success today while also having the adaptability to achieve success tomorrow. In order to be sure that $21^{\text {st }}$ century skills are absorbed, applied, and adapted, the industry must first encourage and identify those who want $21^{\text {st }}$ century skills, those who want to learn and teach others, those who want to actively navigate their own professional course and not merely bob to stay afloat in the wake of another's. The industry, its companies, and its leaders must encourage, identify, and sustain those who are lifelong learners. International Maritime Organization (IMO) regulations and codes and shipboard technologies will always evolve. Lifelong learners will not have perfected tomorrow's skill set today, but they will be in a more advantageous position to be adaptable and successful in the face of these and other changes. It is not always easy, or even possible, to predict the equipment that will drive tomorrow's communications, the safety and environmental regulations that will necessitate tomorrow's compliance, or the platform that will host tomorrow's training. It is reasonable, however, to assert that coupling the continuous acquisition of $21^{\text {st }}$ century skills with the engagement and motivation present in lifelong learners will yield benefits for professional sustainability and adaptability, for both individual and industry gain.

\section{References}

Buchmann, A. (2021, April 13). How COVID-19 has driven a technology revolution in shipping. Marine Log. https://www.marinelog.com/shipping/how-covid-19-has-driven-atechnology-revolution-in-shipping/.

Busteed, B. (2020, February 17). The really good and really bad news on lifelong learning. Forbes. https://www.forbes.com/sites/brandonbusteed/2020/02/17/the-really-good-and-reallybad-news-on-lifelong-learning/\#56c34a911f2c.

Coleman, J. (2017, February 7). Lifelong learning is good for your health, your wallet, and your social life. Harvard Business Review. https://hbr.org/2017/02/lifelong-learning-is-goodfor-your-health-your-wallet-and-your-social-life.

Jurenka, R., Starček, N., Vraňaková, N., \& Cagáňov á, D. (2018). The learning styles of the generation group $\mathrm{Z}$ and their influence on learning results in the learning process. 16th IEEE International Conference on Emerging eLearning Technologies and Applications, November 15-16, Slovakia.

Laal, M. (2011). Lifelong learning: What does it mean?. Procedia - Social and Behavioral Sciences 28 (2011) 470 - 474. 10.1016/j.sbspro.2011.11.090.

National Education Association. (n.d.). 21st century skills. http://www.nea.org/home/34888.htm.

Saavedra, A. R., \& Opfer, V. D. (2012, October 19). Nine lessons on how to teach 21 st century skills and knowledge. Rand. https://www.rand.org/blog/2012/10/nine-lessons-on-how-toteach-21st-century-skills-and.html. 
Smith, J. \& Spurling, A. (1999). Lifelong learning: Riding the tiger. London: Cassell.

SUNY Maritime College. (n.d.). Executive summary: SUNY Maritime College. https://www.sunymaritime.edu/about/strategic-planning/executive-summary.

teAchnology. (n.d.). Letter L Teaching Terms. Terms in teaching that start with letter 1. https://www.teach-nology.com/glossary/terms/1/.

Quast, L. (2014, April 28). Career changers: 4 tips to determine if your skills are transferable. Forbes. $\quad$ https://www.forbes.com/sites/lisaquast/2014/04/28/career-changers-4-tips-todetermine-if-your-skills-are-transferable/\#1a86890616e6.

Vincx, M., Antia, A., Deprez, T., Fiksen, Ø., Koski, M., Mackenzie, B., McGrane, P., O'Carroll, C., Olsen, J., Menezes Pinheiro, L., Ribera d'Alcalà, M., Roullet, G. (2018). Training the 21st century marine professional: A new vision for marine graduate education and training programmes in Europe. Kellett, P., Larkin, K., Heymans, J. J., McDonough, N., Wouters, N., Chu, N-C. [Eds.] Future Science Brief 2 of the European Marine Board, Ostend, Belgium. $47 \mathrm{pp}$. 